\section{THE SYNDROME OF INTESTINAL PSEUDO-OBSTRUCTION}

\author{
BY
}

FREDERICK O. STEPHENS, M.B., F.R.C.S.Ed.

Senior Lecturer in Surgery, University of Sydney; Formerly Senior Registrar, Professorial Surgical Unit, Royal Infirmary, Aberdeen

[With Special Plate]

Most surgeons can recall patients presenting with a condition simulating intestinal obstruction who were found on closer investigation or at laparotomy to have no evidence of organic bowel obstruction. The patients are usually elderly and often have all the classic signs of intestinal obstruction, including colicky abdominal pain, constipation, abdominal distension, obstructive bowel sounds, and the $x$-ray appearance of distended gut with fluid levels; vomiting may also be a feature. The condition is frequently associated with renal or cardiac failure, pneumonia, or an acute infection (particularly of abdominal organs); it may occur after hip or back injuries, and in recent years has been known to follow the use of ganglion-blocking drugs; but occasionally there is no evidence of any associated or predisposing condition. Krippaehne (1961) has seen the syndrome in association with ulcerative colitis and adrenal insufficiency.

Though it is well known to physicians and surgeons alike, there is remarkably little to be found in the literature about this condition. Dudley et al. (1958) reported a series of 13 cases treated at the Edinburgh Royal Infirmary. They applied the term "intestinal pseudo-obstruction," which probably included the condition previously referred to as spastic ileus (Murphy, 1896 ; Aird, 1957 ; Macfarlane and Kay, 1949).

The syndrome recently described by Naish et al. (1960) and called by them " intestinal pseudo-obstruction with steatorrhoea " is probably a separate entity having the specific features of steatorrhoea and certain pathological findings in the musculature of the intestinal wall.

Because these cases not infrequently present a problem in diagnosis and management the following four case histories of patients treated at the Royal Infirmary, Aberdeen, are reported in order to study any lessons which may be learned.

\section{Case Reports \\ Case 1}

A retired labourer aged 72 complained of constipation, colicky lower abdominal pain. and vomiting for five days before admission. Four months previously he had had a similar though less severe attack, but on that occasion symptoms had settled after several large doses of cascara. Investigations after that attack, including stool toluidine test for blood. sigmoidoscopy, and barium enema, were all negative. His urinary stream had been getting increasingly poor. He was known to have had hypertensive heart disease for two years. Symptoms of left ventricular failure had improved with treatment. He was obese, and there was some abdominal distension, particularly in the right iliac fossa. There was slight tenderness over the abdomen, especially on the right side. No masses were felt. Abdominal radiographs showed distended large and small gut with fluid levels.

A diagnosis of subacute intestinal obstruction was made, but at laparotomy no abnormality was found. At the end of the operation the blood urea was $100 \mathrm{mg}$. per $100 \mathrm{ml}$. and on the fourth day was $90 \mathrm{mg}$. per $100 \mathrm{ml}$. The patient made an uneventful recovery, but several months after operation he still had evidence of impaired renal function in that he could concentrate urine only to specific gravity 1017 and had a blood urea of $50 \mathrm{mg}$. per $100 \mathrm{ml}$.

He was last seen two years after operation and has had no further abdominal symptoms. Apart from some dyspnoea on exertion he remains well.

\section{Case 2}

A retired farm worker aged 71 had an attack of diarrhoea which lasted for one day, followed by colicky abdominal pains, constipation, vomiting, and increasing abdominal distension for three days before admission. An enema given before admission produced a poor result. He had no urinary symptoms. Abdominal distension was marked. but no masses were felt. The rectum was empty. Bowel sounds were frequent and high-pitched. Abdominal radiographs showed distended large and small gut with fluid levels (Special Plate, Fig. 1).

A diagnosis of large-bowel obstruction was made and laparotomy performed. The whole of the small gut was grossly distended; the caecum, ascending colon, and proximal transverse colon were distended to a less extent. The distension diminished gradually along the transverse colon, and the distal large bowel was normal. No obstructive lesion or any other abnormality was detected. The bowel was decompressed and the wound closed.

On the day after operation blood urea was $336 \mathrm{mg}$. per $100 \mathrm{ml}$. and serum potassium $6.45 \mathrm{mEq} / \mathrm{l}$. He was treated with a high fluid intake and made a good recovery. On the IIth day the wound looked healthy and sutures were removed, but on the next day the wound burst and was resutured. Convalescence was thereafter uneventful. He was advised to continue a high fluid intake at home, and two months later blood urea was $45 \mathrm{mg}$. per $100 \mathrm{ml}$. Six months after operation urea-clearance tests, intravenous pyelography, and urine concentration and dilution tests gave results within normal limits. He was able to concentrate his urine to a specific gravity of 1022 .

The patient was last seen eight months after operation ; he remained well and had had no further abdominal symptoms.

\section{Case 3}

A 73-year-old retired policeman developed attacks of central abdominal pain and yomiting eight weeks before admission. He also had alternating constipation and diarrhoea. and one week before admission he had a more severe attack of central abdominal pain with distension, but no vomiting. He had no urinary symptoms.

The patient was obese and his abdomen was very distended, with tenderness and some guarding over the right side. No masses were felt. Bowel sounds were present. On rectal examination distended loops of gut were felt. Stool toluidine test for blood was positive. Abdominal radiographs showed distended large bowel with fluid levels rather resembling caecal volvulus (Special Plate, Fig. 2). Blood urea was $80 \mathrm{mg}$. per $100 \mathrm{ml}$., serum amylase and liver function tests were normal. and a rectal tube produced only a little flatus. Sigmoidoscopy was negative.

A diagnosis of intestinal pseudo-obstruction was made, and symptoms slowly subsided with conservative management. Results of barium-enema $x$-ray studies were within normal limits.

On the eighth day after admission the patient was very well and allowed to go home. Three weeks later pain recurred and a transient jaundice developed. Subsequent radiographs indicated a non-functioning gall-bladder. At operation an empyema of the gall-bladder was found and cholecystectomy with drainage of the common bile-duct performed. No other abnormality was detected.

Post-operatively there was a further appearance of bowel obstruction with abdominal distension and high-pitched 
bowel sounds, and radiography showed dilated gut with fluid levels. This episode was associated with reduced urinary output, a blood urea rising to $163 \mathrm{mg}$. per $100 \mathrm{ml}$. on the 12th post-operative day, with serum potassium 5.88 $\mathrm{mEq} / \mathrm{l}$. With the eventual return of normal urinary output, blood chemistry slowly returned to normal and symptoms subsided. The patient was advised to continue with a high fluid intake after discharge from the ward.

He was last seen six months after operation. He was in good health and his blood urea was $30 \mathrm{mg}$. per $100 \mathrm{ml}$., but he had some impairment of renal function as indicated by a maximal concentration capacity to specific gravity 1018 .

\section{Case 4}

A baker aged 66 began to develop abdominal pains, constipation, and abdominal distension five days before admission to hospital. He complained of anorexia, but had not vomited. Previously he had had regular bowel habits and no abdominal symptoms. He had not been losing weight. For eight years he had been taking digitalis for auricular fibrillation with some evidence of cardiac failure.

The patient was breathless, with slight cyanosis and a pronounced cough. His abdomen was distended, but there was no abdominal tenderness and no masses were felt. Frequent high-pitched bowel sounds were heard and there were moist rales over both lungs. A stool toluidine test gave a negative result. Abdominal radiographs showed distended large gut with fluid levels (Special Plate. Fig. 3). Blood urea was $54 \mathrm{mg}$. per $100 \mathrm{ml}$. and plasma $\mathrm{CO}_{2} 66$ vols. \%. An electrocardiogram was reported on as showing "auricular fibrillation with digitalis effects."

A diagnosis of large-bowel obstruction was made, but at laparotomy, though transverse colon, ascending colon, and distal small bowel were very dilated, no organic cause for obstruction or any other abnormality was found. The bowel was deflated and the wound closed.

There was a transient rise in blood urea to $115 \mathrm{mg}$. per $100 \mathrm{ml}$. on the second day, but falling again to $42 \mathrm{mg}$. per $100 \mathrm{ml}$. on the fourth day. He made an uneventful postoperative recovery. He was readmitted one month later for large-bowel radiography and renal function tests, but no significant abnormality was found. He was last seen four years after operation, when. though he continued to have treatment for his cardiac condition, he remained otherwise well and had had no recurrence of the abdominal symptoms.

\section{Discussion}

In an analysis of 13 cases of intestinal pseudoobstruction, Dudley et al. (1958) considered that they fell into three clinical groups: Group I-those secondary to known disease; Group II-primary pseudo-obstruction, cause unknown; and Group III -cases in which prolonged hypotension or hypoxia has been present. Though, in retrospect, at least two of those 13 patients must have had considerable renal functional deficiency (Cases 2 and 3 ), there is no evidence to indicate how many of the remainder may have had renal impairment.

Of the four cases here presented, in Case 1 there was clear evidence of impaired renal function. Also the patient was hypertensive and had attacks of paroxysmal dyspnoea, indicating left ventricular failure. Apart from these associated conditions no definite factor could be implicated to account for the intestinal symptoms.

In Case 2 there was also evidence of severe impairment of renal function. Four days before admission the patient had had diarrhoea. Dehydration probably contributed to the rise in blood urea and serum potassium levels.
In Case 3 there were two " obstructive" episodes with biochemical evidence of renal impairment on each occasion. The first episode may have been precipitated by acute gall-bladder disease, and the second episode followed a major surgical procedure.

It seems likely that the symptoms of intestinal pseudoobstruction in these three patients were associated with impaired renal function. By what mechanism this causes symptoms and signs of intestinal obstruction is not clear, but it may be due to a neuromuscular dysfunction associated with electrolyte disturbances, as has been discussed by Mellinkoff (1959). The exact nature of the mechanism is not known, but symptoms may be associated with either a raised or lowered serum potassium level or with other complex electrolyte disturbances due to renal, hepatic, or metabolic diseases.

In Case 4 there was no evidence of renal failure, but there was severe cardiovascular and respiratory impairment. Possibly this patient could be placed among the group with prolonged hypotension or hypoxia. Again, there is no clear understanding of any association between prolonged hypoxia and a disturbance of neuromuscular function of the alimentary tract.

What lessons may be learned to help differentiate between intestinal pseudo-obstruction and organic bowel obstruction?

First, blood chemistry, including blood urea concentration, should be investigated in any subacute or chronic bowel obstruction, particularly in older patients. This is probably already a routine in most hospitals. The results, however, are not diagnostic of pseudo-obstruction, as it is obvious that a patient with renal failure may still develop an organic bowel obstruction.

Review of the $x$-ray films in retrospect in Case 2 showed there was no "cut-off" of gas in the colon; in fact, there is evidence of the presence of gas all the way to the lower rectum. This finding was also observed by Dudley et al. in some of their cases.

The stool toluidine test for blood may give an indication, but is notoriously unreliable as a diagnostic feature. It was negative in Cases 1,2 , and 4 , but positive in Case 3. A negative finding may possibly be significant in cases of doubt.

It should be noted that there are many conditions other than organic intestinal obstruction or intestinal pseudo-obstruction which may on occasion be associated with abdomınal pains, constipation, and the presence of bowel sounds, with $x$-ray appearances of distended gut and fluid levels. These include-to mention just a few-vascular lesions of gut, lead poisoning, porphyria, morphine overdosage, acute pancreatitis, acute cholecystitis or appendicitis, and acute dysentery. Also these findings may be present after abdominal operations or the administration of an enema, and, of course, fluid levels as seen radiologically may be a feature of paralytic ileus, but bowel sounds are either absent or diminished. However, each of these conditions has other diagnostic features. The $x$-ray appearances of these and other conditions which may show fluid levels on radiological examination have been described by Frimann-Dahl (1951).

\section{Management}

Should a diagnosis be made pre-operatively, the problem then becomes one of management. It would 
seem wise to avoid operations in these apparently poorrisk patients, if possible, though it would appear from our limited experience that the risk of operation is perhaps not so great as is often supposed. All three cases subjected to laparotomy improved quickly after the operation, and the remaining patient withstood subsequent operation well. Undoubtedly, the fluid and electrolyte therapy played the major part in the patients' improvement, but it may be that the rapid and efficient deflation of the dilated bowel at laparotomy helped break a possible vicious circle. In reporting eight similar cases which they labelled "ileus of the colon," Morton et al. (1960) described two patients in which the distended caecum ruptured and two others considered to be in imminent danger of caecal rupture. They point out the value of diagnostic barium-enema examinations, but stress its danger if perforation of the caecum is imminent. They recommend emergency laparotomy when there is gross distension and tenderness in the lower right quadrant. Byrne (1960) has reported the occurrence of caecal and sigmoid volvulus as a sequel to paralytic ileus, and Krippaehne (1961) has also seen patients with intestinal pseudo-obstruction progress to a frank caecal volvulus or diastatic rupture, and it does appear that this could have been the outcome in Case 3 (see Special Plate, Fig. 2) if the bowel had not been effectively deflated. The latter author would then recommend laparotomy where conservative methods of bowel deflation are not rapidly effective. He also recommends the use of a dilute barium enema as of diagnostic value as well as of therapeutic value in helping to deflate the bowel.

One would suggest, therefore, that in established uncomplicated cases the plan of management should be conservative in the first instance, with restoration of fluid and electrolyte balance, gastric aspiration (or deflation by a long intestinal tube may be attempted by surgeons experienced in this method), rectal lavage, and treatment of any associated or predisposing conditions. However, it would seem that operative treatment may not be as hazardous as previously supposed, and it might be recommended under the following conditions: (a) if the diagnosis is in doubt (this will probably still be true of the majority of cases); (b) when there may be an associated condition requiring surgical treatment ; $(c)$ if gross abdominal distension is causing respiratory embarrassment ; $(d)$ when there is a possibility of caecal volvulus, diastatic rupture, or other complication of distended gut; or (e) when efficient conservative management has failed.

\section{Conclusion}

It appears that one form of intestinal pseudoobstruction may be precipitated by an acute dehydrating episode in a patient with poor renal reserve. Once established, there begins a vicious circle of dehydration $\rightarrow$ renal failure $\rightarrow$ uraemia with electrolyte disturbance $\rightarrow$ intestinal pseudo-obstruction $\rightarrow$ vomiting $\rightarrow$ further dehydration. This circle can best be broken by treating any associated or precipitating factors, taking active measures to relieve dehydration and electrolyte disturbance (perhaps including dialysis), and making attempts to deflate the distended gut.

While it is generally agreed that the possibility of intestinal pseudo-obstruction should be borne in mind with a view to avoiding an unnecessary and perhaps hazardous laparotomy, particularly in the elderly or patients with renal failure who present with symptoms and signs of intestinal obstruction, it is inevitable that, in view of present limited knowledge of the exact nature of the condition, the final diagnosis will often not be established until exploratory laparotomy has been performed. Furthermore, it may be that under presentday management laparotomy may not be as dangerous as it is sometimes thought to be, and should in certain circumstances be recommended even in cases where the diagnosis is established.

\section{Summary}

The syndrome of intestinal pseudo-obstruction is described and four cases are presented. On analysis, three of the patients had positive evidence of incipient renal failure. An attack of diarrhoea in one case and acute cholecystitis in another may have been responsible for precipitating a crisis in patients with poor renal reserve. The fourth patient suffered from severe cardiorespiratory deficiency.

The condition should be considered in the differential diagnosis of bowel obstruction, particularly in the elderly or in patients with evidence of renal failure. Findings which may help in making a diagnosis are evidence of renal failure (raised blood urea and serum potassium levels), a negative stool toluidine test for blood, and $x$-ray evidence of the presence of gas in the large bowel right down to the rectum. A dilute barium enema may be of both diagnostic and therapeutic value in certain cases.

Lines of treatment, including the possible place of laparotomy, are discussed.

My thanks are due to the surgeons of Aberdeen Royal Infirmary-Professor W. Wilson, Mr. S. Davidson, Mr. P. Jones, Mr. J. Kyle, and Mr. G. E. Mavor-who allowed me to report the histories of patients under their care. I am grateful to Mr. H. A. F. Dudley, who first stimulated my interest in this subject, and to Dr. M. C. McLeod for advice on the construction of this paper. Since being in the United States of America my interest has been further encouraged by Dr. J. E. Dunphy, whose help and advice I gratefully acknowledge.

\section{REPERENCES}

Aird, I. (1957). A Companion in Surgical Studies, 2nd ed. Livingstone, Edinburgh.

Byrne, J. J. (1960). Amer. J. Surg., 99, 168.

Dudley, H. A. F. Sinclair, I. S. R., McLaren, I. F, McNair, T. J., and Newsam, J. E. (1958). J. roy. Coll. Surg. Edinb., 3, 206 .

Frimann-Dahl, J. C. (1951). Roentgen Examinations in Acute Abdominal Diseases. Blackwell, Oxford.

Krippaehne, W. (1961). Personal communication.

Macfarlane, J. A., and Kay, S. K. (1949). Brit. med. J., 2, 1267.

Mellinkoff, S. M.' (1959). Amer. J. dig. Dis., 4, 653.

Morton, J. H., Schwartz, S. I., and Gramiak, R. (1960). A.M.A. Arch. Surg., 81, 425.

Murphy, J. B. (1896). J. Amer. med. Ass., 26, 15.

Naish, J. M., Capper, W. M., and Brown, N. J. (1960). Gut, 1.

A dozen sheepskins are to be given to the North Canterbury Hospital Board by the New Zealand Wool Board for use in preventing bedsores in the geriatric wards of the Cashmere Sanatorium. The sheepskins are being specially tanned by a Christchurch firm to specifications worked out by the Australian Commonwealth Scientific and Industrial Research Organization. The pelts are being treated in a way which, it is hoped, will enable them to stand up to routine sterilization, including boiling. The leather must also be very soft. Several types of wool are being tried, mostly halfbred varieties. 


\section{F. O. STEPHENS: SYNDROME OF INTESTINAL PSEUDO-OBSTRUCTION}
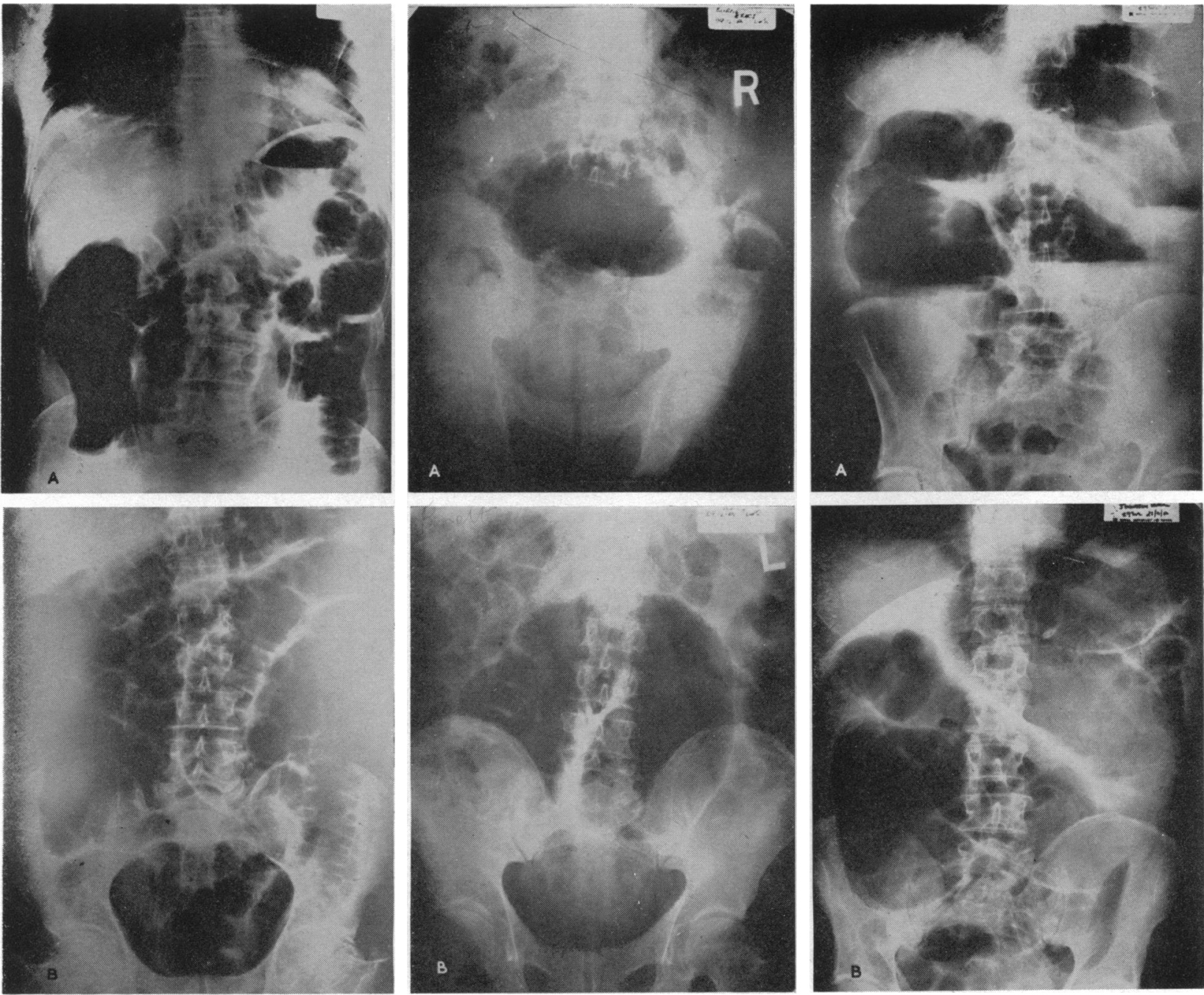

FIG. 1.-(A) Erect and (B) supine abdominal radiographs from Case 2 on large bowel with fluid levels, but gas can be seen extending all the way down to



Fig. 2.-(A) Erect and (B) supine abdominal radiographs from Case 3 on admission, showing dilated large bowel with fluid levels. The appearance is suggestive of caecal volvulus.

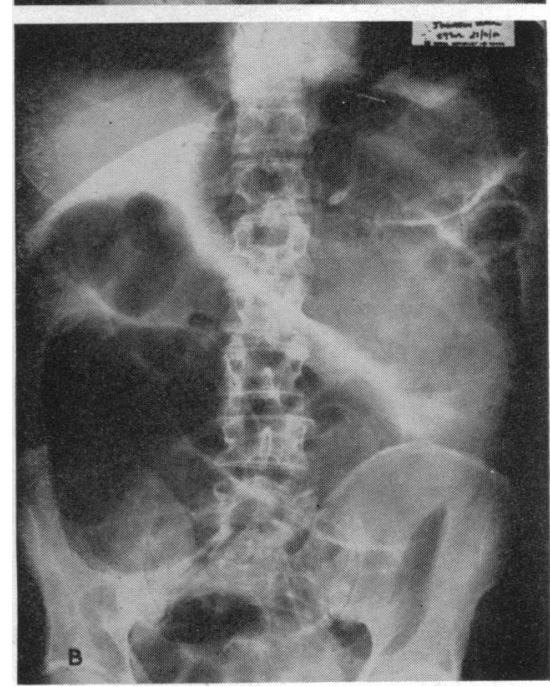

FIg. 3.-(A) Erect and (B) supine abdominal radiographs from Case 4 on admission, showing grossly dilated large bowel with fluid levels.

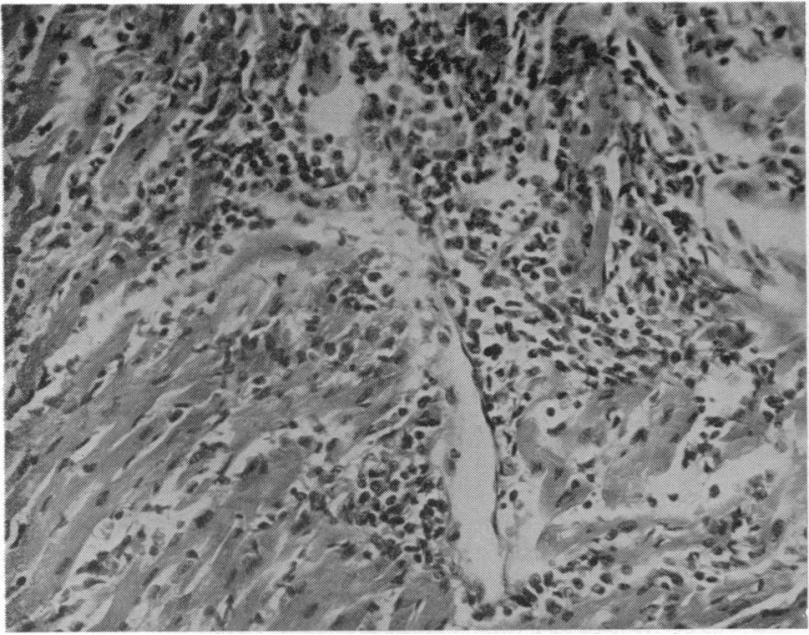

FIg. 1.-Myocardium, showing an inflammatory focus consisting of histiocytes and lymphocytes with an occasional polymorph. $(\mathrm{H}$. and $\mathrm{E}$. $\times 188$.)

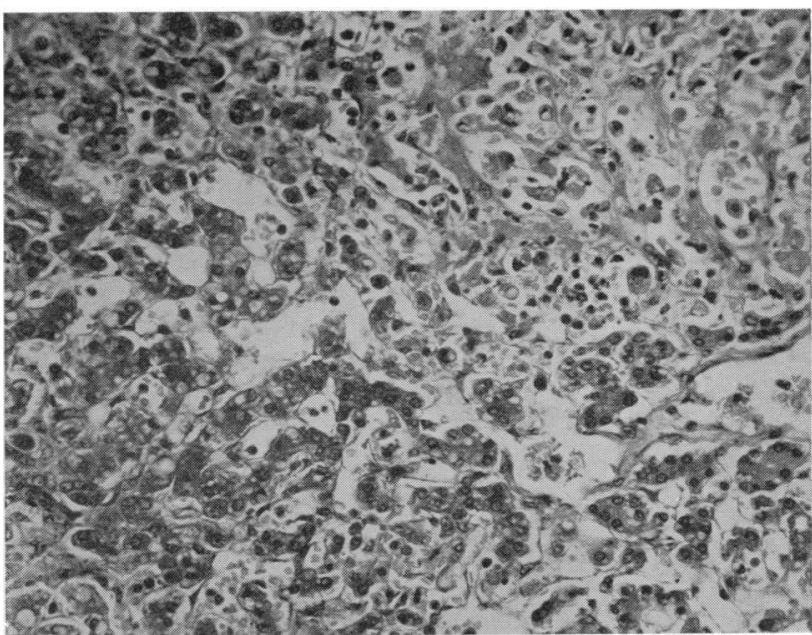

Fig. 2.-Liver. A relatively pale area in the top right-hand quadrant is a focus of necrosis of hepatic cells. (H. and $E$. $\times 188$.) 